\title{
Evaluation of Cyclin Expression in Testicular Germ Cell Tumors: Cyclin E Correlates with Tumor Type, Advanced Clinical Stage, and Pulmonary Metastasis
}

\author{
Milton W. Datta, M.D., Andrew A. Renshaw, M.D., Anindya Dutta, M.D., Ph.D., \\ Michael A. Hoffman, M.D., Kevin R. Loughlin, M.D. \\ Department of Pathology, Medical College of Wisconsin (MWD), Milwaukee, Wisconsin, and Departments \\ of Pathology (AAR, AD) and Urology (MAH, KRL), Brigham and Women's Hospital, Boston, Massachusetts
}

The measurement of proliferative index has yielded promising yet conflicting results in the evaluation of testicular tumors. We have examined the role of Ki-67, along with the cyclins $A$ and $E$ in testicular tumorigenesis. We compared the immunoreactivity of 20 pure seminomas with 20 mixed germ cell tumors composed predominantly of embryonal carcinoma with a variety of proliferation markers, including Ki-67, cyclin A, and cyclin E. All 40 tumors stained for Ki-67, and 19 of 20 (95\%) seminomas and 18 of $20(90 \%)$ embryonal carcinomas stained positively for cyclin A. Cyclin E stained 14 of 19 (74\%) of the embryonal carcinomas and only 4 of 20 (20\%) of the seminomas (Fisher's exact two-tailed test, $P=.0012)$. There was a trend toward larger tumor size for cyclin $\mathrm{E}$-positive seminomas (median, $5.92 \mathrm{~cm}$ versus $3.96 \mathrm{~cm} ; P=.08$ ), although the same correlation was not significant in embryonal carcinomas. For both seminomas and embryonal carcinomas, staining with cyclin $\mathrm{E}$ did not correlate with the presence of lymphovascular invasion or capsular invasion. However, patients who had cyclin E-positive tumors presented with higher clinical stage $(P=.0015)$. In addition, pulmonary spread in embryonal carcinomas (four patients) and seminomas (one patient) occurred only in patients whose tumors were cyclin $\mathrm{E}$ positive $(P=.014)$. Although Ki-67 and cyclin A offer little prognostic information in testicular germ cell tumors, cyclin $\mathrm{E}$ immunoreactivity correlates with tumor type and is strongly predictive of distant tumor spread.

Copyright (C) 2000 by The United States and Canadian Academy of Pathology, Inc.

VOL. 13, NO. 6, P. 667, 2000 Printed in the U.S.A

Date of acceptance: December 10, 1999.

Address reprint requests to: Milton W. Datta, M.D., Department of Pathology, Medical College of Wisconsin, P.O. Box 26509, Milwaukee, WI 53226-0509; e-mail: mdatta@mcw.edu; fax 414-257-7815.
KEY WORDS: Cyclin A, Cyclin E, Germ cell tumor, Immunohistochemistry, Ki-67, Testis.

Mod Pathol 2000;13(6):667-672

The use of proliferation markers for the evaluation and prognosis of germ cell tumors of the testis has not been examined in detail. Most previous studies focused on Ki-67 as a marker of proliferation in germ cell tumors with mixed results (1-3). The cyclins compose a family of cell cycle regulatory proteins that control cellular progression through the cell cycle by regulating the signaling cascade of phosphorylation through cyclins/cyclin-dependent kinases and leading to the phosphorylation of the retinoblastoma protein, its uncoupling from the transcription factor E2-F, and the activation of cellular transcription of genes involved in DNA synthesis and cell division (Fig. 1) (4). The mutation of specific cell cycle proteins such as cyclin D1 in mantle cell lymphoma has implicated specific cyclins in oncogenesis (5). Subsequent studies have correlated dysregulation of cyclins in various solid tumors (6-8). Cyclins, particularly cyclins $\mathrm{A}$ and $\mathrm{E}$, are responsible for cell progression through $S$ phase and DNA synthesis (4). Because cells in S phase are prime targets for DNA damaging agents such as chemotherapy and radiotherapy, an understanding of cyclins A and E expression may help to determine sensitivity to conventional oncologic treatment. The role of these cyclins in testicular germ cell tumors has not been well studied. This article examines the expression of the specific cell cycle regulatory proteins cyclin A and cyclin E, along with Ki-67, in seminomatous and nonseminomatous germ cell tumors of the testis and correlates them with known prognostic markers and patient outcome.

\section{MATERIALS AND METHODS}

\section{Testicular Case Selection}

Germ cell tumors were identified from radical orchiectomies performed at Brigham and Women's 


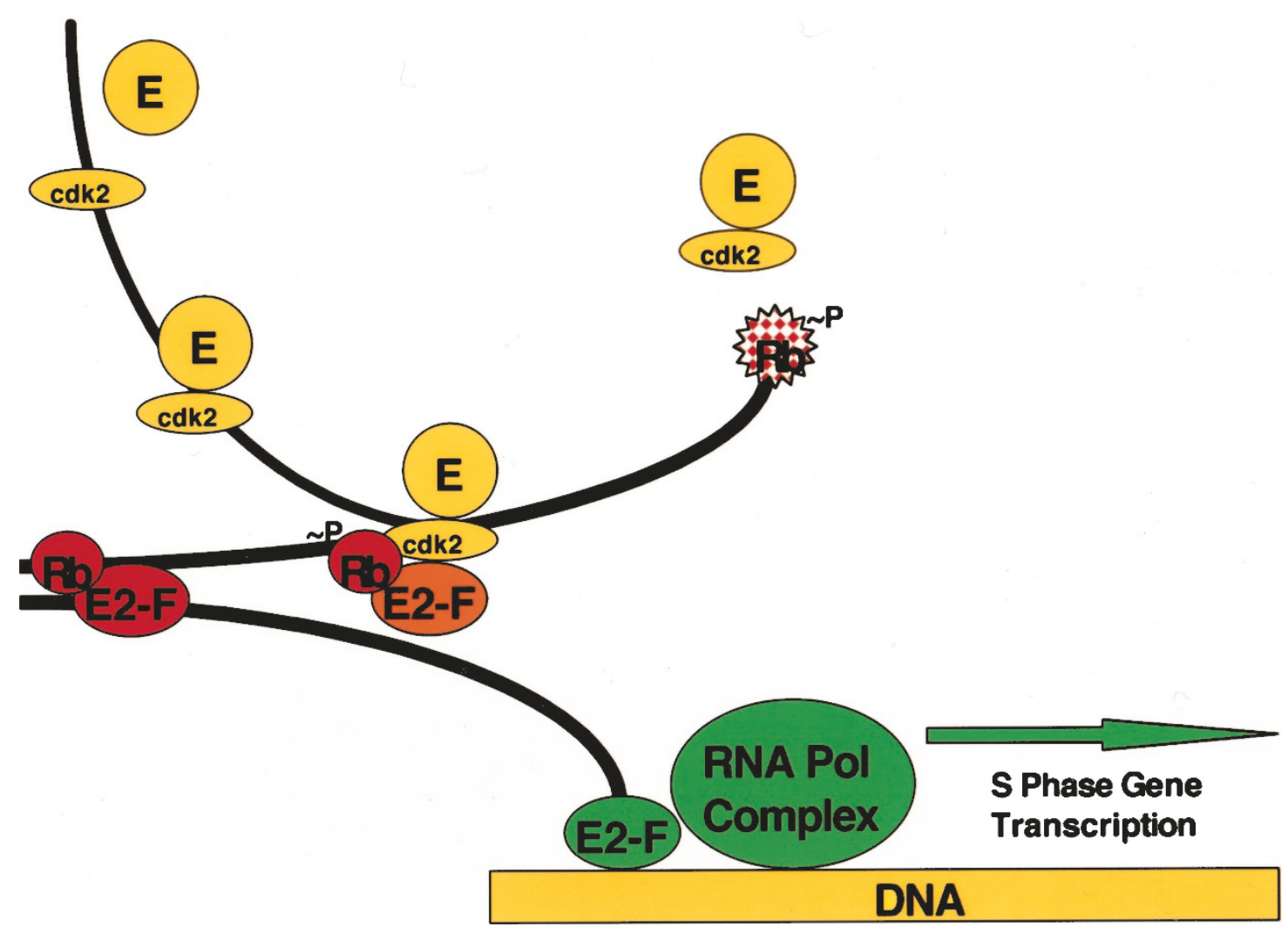

FIGURE 1. Molecular control of the cell cycle. Increasing levels of cyclin E in conjunction with the cyclin-dependent kinase-2 (cdk2) leads to the phosphorylation of the retinoblastoma gene product $(\mathrm{Rb})$. Phosphorylated Rb releases the transcription factor E2-F, allowing E2-F to bind to DNA and in conjunction with RNA polymerase initiate the transcription of S phase-specific genes and entry into S phase.

Hospital between 1988 and 1997. Twenty pure seminomas and 20 mixed germ cell tumors composed predominantly of embryonal carcinoma were identified, and the diagnosis was confirmed by two independent surgical pathologists (AAR and MWD). Recut blank slides for immunohistochemistry were composed of at least $80 \%$ seminoma or embryonal carcinoma and confirmed on a hematoxylin and eosin-stained section.

\section{Immunohistochemistry}

Immunohistochemistry was performed with antibodies directed against Ki-67 (rabbit polyclonal Mib-1; AMAC Inc., Westbrook, ME; 1:100), cyclin A (rabbit polyclonal antibody H-432; Santa Cruz Biotechnology, Santa Cruz, CA; 1:500), and cyclin E (mouse monoclonal antibody HE12; Santa Cruz Biotechnology; 1:500). The conditions for the immunohistochemistry on paraffin sections have been described $(7,8)$. In brief, antibody was incubated with paraffin sections after microwave pretreatment to expose antigen and visualized using the Vector 3,3'-diaminobenzidine detection system (Vector Laboratories, Burlingame, CA) and counterstained with hematoxylin (Gill's formulation; Fisher Scientific, Springfield, NJ).

\section{Analysis}

Slides were scored for tumor positivity, percentage of positive tumor, percentage of positive tumor cells (four independent areas of greatest staining intensity with 50 cells counted), intensity of staining (0 to 3 scale: 0 , no staining; 1 , weak staining observed over hematoxylin-counterstained chromatin; 2 , moderate staining that partially obscured the counterstained chromatin; 3 , strong staining that completely obscured the counterstained chromatin), and pattern of staining (nuclear, nucleolar, cytoplasmic, membranous). Cyclin and Ki-67 staining was considered positive only if nuclear staining was identified. The presence of nuclear staining in lymphocytes (Ki-67), normal seminiferous tubules (cyclin A), or stromal vessels (cyclin E) has been previously observed (8) and was required as evidence of positive staining in the protocol. Intratubular germ cell neoplasia unclassified was evaluated, when present, for Ki-67, cyclin A, and cyclin E expression with nuclear staining in any identifiable tumor cells classified as positive. Comparison of immunohistochemical reactivity in different subgroups with clinical data was performed using a two-tailed Fisher's exact $t$ test for categorical data with a nonparametric Mann-Whitney $U$ test for continuous data. 


\section{RESULTS}

\section{Tumor and Patient Profiles}

Forty tumors were included in this series, 20 malignant mixed germ cell tumors with the majority component of embryonal carcinoma and 20 seminomas. These arose in men with a mean age of 30.9 years, with seminomas occurring at a mean age of $27 \pm 4.3$ years and embryonal carcinomas presenting at a mean age of $30 \pm 7.1$ years. Tumor size averaged $3.8 \mathrm{~cm}$ (seminomas, $4.3 \pm 3.7 \mathrm{~cm}$; embryonal carcinomas, $3.3 \pm 2.5 \mathrm{~cm})$. Lymphovascular invasion was present in 19 of 40 cases (11 embryonal carcinoma and 8 seminoma), and penetration through the tunica by tumor was present in 12 of 40 cases ( 7 embryonal carcinoma and 5 seminoma). Of the 20 embryonal carcinomas, 3 were pure embryonal carcinomas and the remaining 17 were mixed germ cell tumors composed of an average of $80 \%$ embryonal carcinoma (range, 40 to $99 \%$ ) and secondary components including yolk sac tumor and teratoma. One case of embryonal carcinoma was notable for a single focus of choriocarcinoma that was present on additional slides obtained while carrying on this investigation. Intratubular germ cell neoplasia unclassified was present on the slides examined in 17 cases of seminoma and in 16 cases of embryonal carcinoma.

Clinical staging of the tumors and follow-up data are summarized in Table 1. American Joint Commission for Cancer 1997 clinical staging was available on 37 of 40 cases; 5 of 37 patients presented with Stage III disease ( 4 embryonal carcinoma and 1 seminoma patient), and 8 of 37 presented with Stage II disease (6 embryonal carcinoma and 2 seminoma patients). Follow-up clinical data were obtained on 36 of 40 patients with a median of 43 months of follow-up (range, 7 to 120 months). Five patients relapsed (three embryonal carcinoma and two seminoma patients), three regionally and two distantly. None has died of disease.

TABLE 1. Clinical and Pathologic Characteristics of Tumors

\begin{tabular}{lcc}
\hline & Seminoma & Embryonal Carcinoma \\
\hline Number of cases & 20 & 20 \\
Patient age (mean \pm SD) & $27 \pm 4.3$ & $30 \pm 7.1$ \\
Tumor size (mean \pm SD) & $4.3 \pm 3.7$ & $3.3 \pm 2.5$ \\
Lymphovascular invasion present & 8 & 11 \\
Tunica invasion present & 5 & 7 \\
Pulmonary metastasis present & 1 & 4 \\
Clinical Stage I & 17 & 10 \\
Clinical Stage II & 2 & 6 \\
Clinical Stage III & 1 & 4 \\
Relapse & 2 & 3 \\
Follow-up months & & 48 \\
$\quad$ Median & 40 & $10-120$ \\
Range & $7-87$ & \\
\hline
\end{tabular}

Expression of Ki-67 and Cyclin A in Germ Cell

\section{Tumors}

Table 2 and Figure 2 summarize the immunohistochemical results for cyclins $\mathrm{A}$ and $\mathrm{E}$ and Ki-67. Expression of Ki-67 was present in $100 \%$ (20 of 20) of seminomas and $100 \%$ (20 of 20 ) of embryonal carcinomas with a mean of $86 \%$ and $70 \%$ nuclei staining in embryonal carcinomas and seminomas, respectively. In the embryonal carcinomas, 16 of 20 tumors revealed greater than $90 \%$ nuclei staining; the remaining 4 cases ranged between 26 and $64 \%$. For the seminomas, 11 of 20 cases revealed greater than $70 \%$ nuclei staining; the remaining samples ranged between 44 and $68 \%$. Ki-67 staining was noted in intratubular germ cell neoplasia in 14 of 17 (82\%) evaluable seminomas and in 14 of 16 (88\%) evaluable embryonal carcinomas.

Expression of cyclin A was present in 95\% (19 of 20 ) of seminomas and in $90 \%$ (18 of 20) of embryonal carcinomas. Of note, embryonal carcinomas tended to have a higher intensity of staining (average, 2.1 versus 1.0 ; scale, 0 to 3 ) and a greater number of positive cells ( $66.5 \%$ versus $21.9 \%$ ) than the corresponding seminomas, although these numbers did not achieve statistical significance. The extent of cyclin A and Ki-67 expression in the tumors was strongly correlated. Cyclin A was present in intratubular germ cell neoplasia in 13 of $16(81 \%)$ evaluable embryonal carcinomas but in only 7 of $17(41 \%)$ evaluable seminomas $(P=.03)$.

\section{Expression of Cyclin E in Germ Cell Tumors}

Cyclin E expression was present in 14 of 19 (74\%) of the embryonal carcinomas and in only 4 of 20 (20\%) of the seminomas examined $(P=.0012)$. When expression was present, there was no significant difference in intensity of staining (1.5 versus 1.0 ) or percentage of cells staining ( $26 \%$ versus $44 \%$ ) in embryonal carcinomas versus seminomas respectively.

There was a trend between expression of cyclin $\mathrm{E}$ in seminomas and increased tumor size $(3.96 \mathrm{~cm}$ median size in negative tumors versus $5.92 \mathrm{~cm}$ median size in positive tumors; $P=.08$ ), but cyclin $\mathrm{E}$ did not correlate with the presence of capsular invasion $(P=.5)$, vascular invasion $(P=1.0)$, or patient age $(P=.66)$. No correlations were noted in the embryonal carcinomas for size $(P=.90)$, capsular invasion $(P=1.0)$, vascular invasion $(P=.13)$, or patient age $(P=.3)$. Cyclin $\mathrm{E}$ expression in intratubular germ cell neoplasia was present in only 2 of 17 evaluable seminomas (12\%) and in none of the 16 evaluable embryonal carcinomas. When present in the seminomas, intratubular germ cell neoplasia cyclin E staining correlated with cyclin E expression in the associated invasive tumor. The interpretation of cyclin $\mathrm{E}$ staining in intratubular 


\begin{tabular}{|c|c|c|c|c|c|c|}
\hline & \multicolumn{3}{|c|}{ Seminoma } & \multicolumn{3}{|c|}{ Embryonal Carcinoma } \\
\hline & Ki-67 & Cyclin A & Cyclin E & Ki-67 & Cyclin A & Cyclin E \\
\hline Number of cases & 20 & 20 & 20 & 20 & 20 & 19 \\
\hline Number positive & 20 & 19 & 4 & 20 & 18 & 14 \\
\hline Tumor positivity (\%) & 100 & 95 & 20 & 100 & 90 & 74 \\
\hline Cellular positivity (\%) & 70 & 22 & 44 & 86 & 66 & 26 \\
\hline Staining intensity (0-3) & 3.0 & 1.0 & 1.0 & 3.0 & 2.1 & 1.5 \\
\hline
\end{tabular}

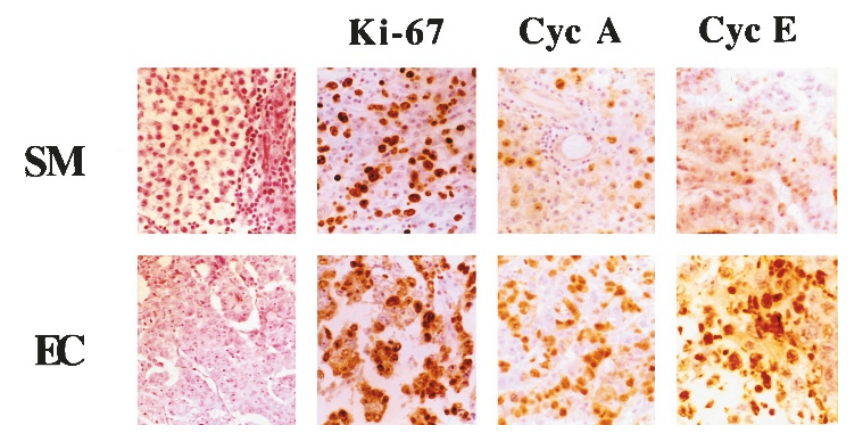

FIGURE 2. Cyclin expression in germ cell tumor seminomas (SM): hematoxylin and eosin-stained section, Ki-67, cyclin A, cyclin E. Embryonal carcinomas (EC): hematoxylin and eosin-stained section, Ki-67, cyclin A, cyclin E $(400 \times)$. Note the strong nuclear staining in both Ki-67 samples. Although nuclear staining is present in both cyclin A samples, stronger staining and more positive cells are present in embryonal carcinomas. Cyclin E nuclear staining is also present in embryonal carcinomas.

germ cell neoplasia was complicated by the presence of strong cytoplasmic staining in normal germ cells.

When examining all the germ cell tumors, seminomas, and embryonal carcinomas, certain correlations with cyclin E positivity and clinical parameters could be drawn (Table 3). Germ cell tumors that were cyclin $\mathrm{E}$ positive were more likely to present with clinical Stage II or III disease $(P=$ .0015). In addition, only tumors that were cyclin $\mathrm{E}$ positive presented with pulmonary metastasis $(P=$ $.014)$.

\section{DISCUSSION}

The use of proliferative activity and cell cycle rate in the analysis of testicular cancer has been attempted in the past with promising results (9). The hope of identifying patients who have limited disease and who would be at risk for recurrent and/or disseminated disease has spurred the examination of multiple prognostic factors. The best prognostic factors include the clinical and pathologic stage, tumor type, percentage of embryonal carcinoma, and the presence of lymphovascular invasion (10, 11). One study suggested that in seminomas, a tumor size of greater than $6 \mathrm{~cm}$ may predict a higher relapse rate (88\% versus $67 \%$ at 5 years) (12). The volume of embryonal carcinoma also has been proposed as a marker for relapse, although it was most effective when used in association with proliferative activity (9). The advent of markers that measure proliferation rate in tumor cells presents a new opportunity for the development of better prognostic markers for tumor relapse. There is evidence that high proliferative activity (as measured by S-phase fraction using flow cytometry) correlates with the presence of metastatic disease in testicular tumors (9). Studies of proliferative activity in testicular tumors have focused on the use of Ki-67 as a tumor marker. Ki-67 is an antigen of $395 \mathrm{kdal}$ that is expressed in all phases of the cell cycle except for $G_{0}$ (resting phase). Three studies have debated the importance of Ki-67 expression in the ability to separate a population of patients who are at high risk for metastatic disease from testicular tumors. Albers et al. (1) found that Ki-67 expression as measured by MIB-1 staining of tumors was significantly higher in patients who had metastatic disease and was a predictor of metastasis. In their study, no patient with a MIB-1 level of less than $52 \%$ had metastasis. Alternatively, Heidenreich et al. (2) showed that total Ki-67 expression was not able to predict pathologic Stage I or Stage II disease any better than percent-

TABLE 3. Ki-67, Cyclin A, and Cyclin E and Tumor Stage

\begin{tabular}{|c|c|c|c|}
\hline & $\begin{array}{c}\text { Ki-67 } \\
\text { (\# positive/total \#) }\end{array}$ & $\begin{array}{c}\text { Cyclin A } \\
\text { (\# positive/total \#) }\end{array}$ & $\begin{array}{c}\text { Cyclin E } \\
\text { (\# positive/total \#) }\end{array}$ \\
\hline Stage I Positive & $27 / 27$ & $25 / 27$ & $9 / 26$ \\
\hline Seminoma & $17 / 17$ & $16 / 17$ & $3 / 17$ \\
\hline Embryonal Carcinoma & $10 / 10$ & $9 / 10$ & $6 / 10$ \\
\hline Stage II Positive & $8 / 8$ & $8 / 8$ & $5 / 8$ \\
\hline Seminoma & $2 / 2$ & $2 / 2$ & $0 / 2$ \\
\hline Embryonal Carcinoma & $6 / 6$ & $6 / 6$ & $5 / 6$ \\
\hline Stage III Positive & $5 / 5$ & $4 / 5$ & $5 / 5$ \\
\hline Seminoma & $1 / 1$ & $1 / 1$ & $1 / 1$ \\
\hline Embryonal Carcinoma & $4 / 4$ & $3 / 4$ & $4 / 4$ \\
\hline
\end{tabular}


age of embryonal carcinoma or the presence of lymphovascular invasion. Hori et al. (3) examined the role of proliferation in seminomas and found that although mitotic index, Ki-67, and proliferating cell nuclear antigen expression were directly correlated with the presence of metastatic disease, there was statistical overlap between the distributions and the control groups, suggesting that Ki-67 activity makes only a small contribution to the overall malignancy of testicular seminomas. Our results confirm the lack of specificity of Ki-67 in the identification of a subset of patients who are at risk for disseminated disease. In our study, there was a uniformly high level of Ki-67 expression as detected by MIB-1 staining in embryonal carcinomas. Patients who had clinical Stage I disease averaged $86 \%$ cellular positivity compared with $88 \%$ for patients who had clinical Stage II or III disease. In addition, one patient who had an MIB-1 staining index of $26 \%$ presented with clinical Stage II disease.

The role of cyclin A and cyclin E in testicular germ cell tumors has not been previously examined. Recent work has elucidated many genes involved in the positive regulation of the eukaryotic cell cycle. These genes are grouped into two main groups: cell cycle-dependent kinases (cdk) and cyclins. The cdks regulate the cell cycle via the phosphorylation of the retinoblastoma gene product, thus allowing the release of the transcription factor E2-F and the transcription of genes needed for DNA synthesis (4) (Fig. 1). The cyclins provide the cell cycle specificity to the cdk phosphorylation signal. The cyclins are transiently produced during various phases of the cell cycle and when present activate the cdks, allowing for progression through the cell cycle. Cyclins are rapidly produced and then promptly degraded by ubiquitin-mediated proteolysis.

Numerous cyclins have been identified, including cyclin A, cyclins D1-3, and cyclin E. Cyclin A is a 60 kdal protein that is involved in DNA replication and mitosis. It is produced and transported to the nucleus during $S$ and $G_{2}$ phases and is degraded after the cell enters the $M$ (mitosis) phase (13). Both cyclins D2 and E are expressed in the $G_{1}$ phase of the cell cycle and allow progression into $S$ phase in a sequential manner. Cyclin D2 is expressed in early $\mathrm{G}_{1}$ with subsequent expression of cyclin E before $\mathrm{S}$ phase entry and DNA synthesis. Cyclin $\mathrm{E}$ is a $45 \mathrm{kdal}$ nuclear protein that appears in late $\mathrm{G}_{1}$ phase and is degraded after the cell enters the $S$ phase and is believed to be the main regulator of entry into $S$ phase and DNA synthesis $(14,15)$. Cyclin E (in our study) was difficult to detect by immunostaining in proliferating cells of normal tissues, although it has been detected in tumor cells from the breast and cervix $(7,8,16)$.
The frequency of cyclin-positive tumor cells $(66 \%$ of seminoma tumor cells and $92 \%$ of embryonal carcinoma tumor cells express either cyclin E or A) is dramatically higher than what has been seen in other cyclin studies of prostate $(6.7 \%)$, breast $(22 \%)$, or kidney tumors $(6.0 \%)$ in which both cyclin A and E have been examined together $(7,8,17)$. These results may indicate that in testicular tumors, more cells are found past the restriction point in $G_{1}$ and already traversing $S$ phase, the phase in which both cyclins A and E would be coexpressed. The restriction point is considered the last possible point to halt cell cycling and entry into S phase and DNA replication. Cells that are before the restriction point and are subjected to DNA damage can halt cell cycle progression and repair their DNA. Cells that are already traversing $S$ phase cannot repair DNA damage (from radiation or chemotherapy), and they undergo cell death. This high fraction of cells already committed to traversing $\mathrm{S}$ phase, as evidenced by the large numbers of cells expressing cyclins A and E, may explain why these tumors have a high sensitivity to radiation- and chemotherapyinduced DNA damage.

Our results show that cyclin E is preferentially expressed in embryonal carcinomas, with decreased expression in seminomas. Studies that have examined the role of cyclin $\mathrm{E}$ in neoplasia have noted its correlation with tumorigenesis in gastric and colonic tumors, along with carcinoma of the renal pelvis and ureter. Yasui et al. (18) found cyclin E expression in $49 \%$ of colonic adenomas and in $59 \%$ of adenocarcinomas and noted a tendency of cyclin $\mathrm{E}$ to be expressed in deeply invasive tumors and lymph node metastases. Furihata et al. (19) noted expression of cyclin $\mathrm{E}$ in $30 \%$ of transitional cell carcinomas of the renal pelvis and showed a relationship of p53 and cyclin E positivity in highgrade tumors. Likewise, Sakaguchi et al. (20) noted a similar relationship with p53 and cyclin E positivity and poor prognosis in gastric carcinomas. The widespread expression of cyclin $\mathrm{E}$ in testicular germ cell tumors is in contrast to what has been noted in prostate and renal tumors but is similar to what has been seen in breast and cervical tumors $(7,8,16,17$, 21 ). It should be noted that the examination of tumors that were chosen for their high percentage of embryonal carcinoma may introduce a selection bias for a more aggressive subset of germ cell tumors. This subset was chosen on the basis of interest in the most clinically relevant cell types in germ cell tumor histology and to reduce confounding variables such as differing percentages of other tumor elements. Although this study design allows the comparison between these two tumor elements, it does not examine the cyclin expression pattern in other germ cell components such as yolk sac tumor, teratoma, or choriocarcinoma. Where 
examined, focal cyclin E was expressed in proliferating epithelial cells of teratomatous elements but was not expressed in yolk sac elements. Of note, high levels of cyclin $\mathrm{E}$ were noted in a single focus of choriocarcinoma present in one case. This association between cyclin E expression with this aggressive germ cell element is intriguing and is under further investigation. The mechanism for cyclin $\mathrm{E}$ expression in testicular tumors remains to be elucidated. Marone et al. (22) noted increased cyclin E expression in ovarian tumors and documented both increased cyclin E RNA transcription and cyclin $\mathrm{E}$ gene amplification as the mechanisms involved in this overexpression.

The expression of cyclin E does not seem to correlate with either cyclin A or Ki-67 expression and seems to be regulated independently of these two markers. Although cyclin E did show a correlation with tumor size in seminomas, this was not true for the embryonal carcinomas. This difference may reflect the differing studies on the correlation between tumor size and clinical outcome in seminomas versus embryonal carcinomas. Tumor size as measured by the single largest dimension has been correlated with recurrence in seminomas (12). That cyclin E correlates with greatest single dimension tumor size and with aggressiveness supports this relationship. In embryonal carcinomas, it has been found that three-dimensional tumor volume correlated with recurrence (9). The lack of correlation between tumor size and cyclin E expressing tumor aggressiveness in embryonal carcinomas may reflect the need for a more detailed tumor volume measurement or the inherently aggressive phenotype of embryonal carcinoma. Our results show that cyclin E expression is strongly correlated with tumor stage and may be necessary for pulmonary tumor dissemination in both seminomas and embryonal carcinomas. All tumors with Stage III disease had cyclin E expression. Not a single case of cyclin E-negative seminoma or embryonal carcinoma spread to the lungs, and only one of six embryonal carcinomas that spread to clinical Stage II disease was cyclin E negative.

We conclude that cyclin $\mathrm{E}$ in particular seems to distinguish a subset of more aggressive tumors and may prove to be a valuable adjunct in determining the relapse rate and tumor progression of testicular germ cell tumors.

\section{REFERENCES}

1. Albers P, Bierhoff E, Neu D, Fimmers R, Wernert N, Muller SC. MIB-1 immunohistochemistry in clinical stage I nonseminomatous testicular germ cell tumors predicts patients at low risk for metastasis. Cancer 1997;79:1710-6.

2. Heidenreich A, Schenkmann NS, Sesterhenn IA, Mostofi FK, McCarthy WF, Heidenreich B, et al. Immunohistochemical expression of Ki-67 to predict lymph node involvement in clinical stage I nonseminomatous germ cell tumors. J Urol 1997;158:620-5.

3. Hori K, Uematsu K, Yasoshima H, Sakurai K, Yamada A. Contribution of cell proliferative activity to malignancy potential in testicular seminoma. Pathol Int 1997;47:282-7.

4. Gillett CE, Barnes DM. Demystified . . c cell cycle. Mol Pathol 1998;51:310-6.

5. Makamura S, Yatabe Y, Seto M. Cyclin D1 overexpression in malignant lymphomas. Pathol Int 1997;47:421-9.

6. Hui AM, Makuuchi M, Li X. Cell cycle regulators and human hepatocarcinogenesis. Hepatogastroenterology 1998;45:163542.

7. Mashal RD, Lester S, Corless C, Richie JP, Chandra R, Propert $\mathrm{KJ}$, et al. Expression of cell cycle regulated proteins in prostate cancer. Cancer Res 1996;56:4159-63.

8. Dutta A, Chandra R, Leiter LM, Lester S. Cyclins as markers of tumor proliferation. Immunocytochemical studies in breast cancer. Proc Natl Acad Sci U S A 1995;92:5386-90.

9. Albers P, Ulbright TM, Albers J, Miller GA, Orazi A, Crabtree $\mathrm{W}$, et al. Tumor proliferative activity is predictive of pathologic stage in clinical stage A nonseminomatous testicular germ cell tumors. J Urol 1996;155:579-86.

10. Moul JW, McCarthy WF, Fernandez EB, Sesterhenn IA. Percentage of embryonal carcinoma and of vascular invasion predicts pathological stage in clinical stage I nonseminomatous testicular cancer. Cancer Res 1994;54:362-4.

11. Heidenreich A, Sesterhenn IA, Mostofi FK, Moul JW. Prognostic risk factors that identify patients with clinical stage I nonseminomatous germ cell tumors at low risk and high risk for metastasis. Cancer 1998;83:1002-11.

12. Warde P, Gospodarowicz MK, Banerjee D, Panzarella T, Sugar L, Catton CN, et al. Prognostic factors for relapse in stage I testicular seminoma treated with surveillance. J Urol 1997;157:1705-10.

13. Desdouets C, Sobczak-Thepot J, Murphy M, Brechot C. Cyclin A: function and expression during cell proliferation. Prog Cell Cycle Res 1995;1:115-23.

14. Sauer K, Lehner CF. The role of cyclin $\mathrm{E}$ in the regulation of entry into S phase. Prog Cell Cycle Res 1995;1:125-39.

15. Keyomarsi K, Herliczek TW. The role of cyclin E in cell proliferation, development, and cancer. Prog Cell Cycle Res 1997;3:171-91.

16. Quade BJ, Park JJ, Crum CP, Sun D, Dutta A. In vivo cyclin E expression as a marker for early cervical neoplasia. Mod Pathol 1998;11:1238-46.

17. Renshaw AA, Loughlin KR, Dutta A. Cyclin A and Mib1 (Ki-67) as markers for proliferative activity in primary renal neoplasms. Mod Pathol 1998;11:963-6.

18. Yasui W, Akama Y, Kuniyasu H, Yokozaki H, Semba S, Shimamoto $\mathrm{F}$, et al. Expression of cyclin $\mathrm{E}$ in human gastric adenomas and adenocarcinomas: correlation with proliferative activity and p53 status. J Exp Ther Oncol 1996;1:88-94.

19. Furihata M, Ohtsuki Y, Sonobe H, Shuin T, Yamamoto A, Terao N, et al. Prognostic significance of cyclin E and p53 protein overexpression in carcinoma of the renal pelvis and ureter. Br J Cancer 1998;77:783-8.

20. Sakaguchi T, Watanabe A, Sawada H, Yamada Y, Yamashita J, Matsuda M, et al. Prognostic value of cyclin E and p53 expression in gastric carcinoma. Cancer 1998;82:1238-43.

21. Scott KA, Walker RA. Lack of cyclin E reactivity in nonmalignant breast and association with proliferation in breast cancer. Br J Cancer 1997;76:1288-92.

22. Marone M, Scambia G, Giannitelle C, Ferrandina G, Masciullo V, Bellacosa A, et al. Analysis of cyclin E and Cdk2 in ovarian cancer: gene amplification and RNA overexpression. Int J Cancer 1998;75:34-9. 